\title{
Postclassical Turbulence Mechanics
}

\author{
Jaak Heinloo \\ Marine Systems Institute, Tallinn University of Technology, Tallinn, Estonia \\ Email: jaak.heinloo@msi.ttu.ee
}

Received January 13, 2013; revised February 15, 2013; accepted February 26, 2013

Copyright (C) 2013 Jaak Heinloo. This is an open access article distributed under the Creative Commons Attribution License, which permits unrestricted use, distribution, and reproduction in any medium, provided the original work is properly cited.

\begin{abstract}
This paper surveys the formalism and applications of the postclassical turbulence mechanics (PCTM) grounded on the characterization of turbulent flow field in infinitesimal surroundings of the flow field points besides the flow velocity at these points also by the curvature of the velocity fluctuation streamlines passing these points. The PCTM applies this step to found the turbulence split into the orientated and the non-orientated constituents. The split specifies the competence of the classical turbulence mechanics (CTM) to the description of the non-orientated turbulence constituent and delegates the description of the orientated turbulence constituent (in the spirit of the theory of micropolar fluids) to the equation of moment-of-momentum. The concurrent presence of the orientated (relatively large scale) and the non-orientated (relatively small scale) turbulence constituents enables to compile the CTM and the conception of L. F. Richardson and A. N. Kolmogorov about the cascading turbulence (RK conception) within a conjoint formalism. The compilation solves the classical conflict between the CTM and the RK conception, though evinces a conflict of another type characterized as paradigmatic.
\end{abstract}

Keywords: Fluid Mechanics; Turbulence; Micropolar Fluids

\section{Introduction}

According to the classical turbulence mechanics (CTM), the flow state in the infinitesimal surrounding of each flow-field point is uniquely characterized by the flow velocity at this point. The postclassical turbulence mechanics (PCTM) modifies this statement by constituting a flow state in the infinitesimal surrounding of each flow point characterized in addition to the flow velocity at this point also by the curvature of the velocity fluctuation streamlines passing this point. The complementation is an outcome of the analysis of the relation between the CTM, the conception of L. F. Richardson [1] and A. N. Kolmogorov [2] (RK conception) about the cascading eddy structure of turbulence and the idea about the applicability of the theory of micropolar fluid (MF) [3-7] to the description of turbulent flows [8-12]. The analysis follows the general principles of the statistical physics connecting the properties of the statistical ensembles to the specific conditions of their formation formulated in average terms $[13,14]$. The adjustment of these principles within the context of the turbulence problem has been explained in [15-21] and summarized in [22] (together with the physical-historical background of the turbulence problem) as the physical doctrine of turbulence (PDT).
The formalism of the PCTM (section 2) utilizes the suggested complemented characterization of the flowfield states in the infinitesimal surroundings of the flowfield points as a precondition for definition of a kinematical-dynamical pair of the Eulerian flow-field characteristics reflecting local average effect of a prevailing orientation of the large-scale turbulence constituent. The turbulence property characterized by the defined quantities founds the decomposition of turbulence into its orientated (relatively large scale) and non-orientated (relatively small scale) constituents, delegates the description of the orientated turbulence constituent (in the spirit of the theory of MF) to the equation of moment-of-momentum, specifies the competence of the CTM to the description of the non-orientated constituent of turbulence and provides an opportunity to reflect the RK conception (in two-scale approximation) in formulation of turbulence mechanics (TM). Besides, the turbulence properties reflected by the defined flow-field characteristics introduce substantial particularization to the description of energetic and transport processes in turbulent media (A more general setup of the PCTM for the multiscale representation of turbulent flow-field can be found in [19-21]).

Section 3 discusses a complementation of the ex- 
plained in the Section 2 all-purpose formalism of the PCTM by the appropriate closure assumptions summing up in the form of the theory of rotationally anisotropic turbulence (the RAT theory) [15,16,23] (henceforth, [23]). The applied closure constitutes the generalized forces driving the motion linearly connected to the respective generalized velocities, which ascribes the coefficients introduced by the closure with a plain and unambiguous physical sense. The perspectives of application of the RAT theory are exemplified in the Section 4 on several examples.

Section 5 (Conclusion) comments the reception of the PCTM (together with the RAT theory and its applications) by the scientific society engaged within the CTM. The reception is characterized as evidencing a substantial paradigmatic conflict between the expressed by the PDT physical look to the turbulence problem and the look to this problem kept by the CTM. The latter reduces the turbulence problem to either a huge number of applied tasks or to the problem of integration of equations of classical fluid mechanics. The aim of the current paper is to motivate physicists to determine their own position in this conflict.

\section{The Formalism of the PCTM}

\subsection{The Grounding Steps of Formulation of the PCTM}

The formalism of the PCTM begins from claiming the momentary states of the turbulent flow-field fixed in infinitesimal surroundings of each flow-field point besides the flow velocity $v$ at this point by the curvature of the velocity fluctuation streamline passing this point. The claim is accompanied with the inclusion of the curvature characteristics of the velocity fluctuation streamlines to the arguments of the probability distribution of the medium motion states at the flow-field points. In the following the PCTM applies this preliminary step as a necessary precondition to determine the dynamical-kinematical pair of the Eulerian flow-field characteristics

$$
\boldsymbol{M}=\left\langle\boldsymbol{v}^{\prime} \times \boldsymbol{R}\right\rangle=\left\langle R^{2} \boldsymbol{e} \times \dot{\boldsymbol{e}}\right\rangle
$$

and

$$
\boldsymbol{\Omega}=\left\langle\boldsymbol{v}^{\prime} \times \boldsymbol{k}\right\rangle=\langle\boldsymbol{e} \times \dot{\boldsymbol{e}}\rangle,
$$

complementing the average flow field characteristics introduced in the CTM. In (1) and (2) (and hereafter): angular brackets denote statistical averaging, $\boldsymbol{v}^{\prime}=\boldsymbol{v}-\boldsymbol{u}$ (in which $\boldsymbol{u}=\langle\boldsymbol{v}\rangle$ ) denotes the fluctuating constituent of the flow velocity; $\boldsymbol{e}=\boldsymbol{v}^{\prime} / v^{\prime}$ (in which $v^{\prime}=\left|\boldsymbol{v}^{\prime}\right|$ ); $\boldsymbol{k}=$ $\partial \boldsymbol{e} / \partial s$ (in which $s$ is the length of the curve of $\boldsymbol{v}^{\prime}$ streamline passing a flow field point) is the curvature vector of the $\boldsymbol{v}^{\prime}$ streamline passing the flow field point; $\boldsymbol{R}=\boldsymbol{k} / k^{2} \quad$ (in which $k=|\boldsymbol{k}|$ ) is the curvature radius vector corresponding to $\boldsymbol{k} ; R=|\boldsymbol{R}|$; and the overdot denotes the full time derivative $\partial / \partial t+v^{\prime} \partial / \partial s$.

The defined in (1) and (2) quantities characterize the average state of motion of Lagrangian particles passing the flow field points while $\boldsymbol{M}$ has the sense of the average density (per unit mass) of the moment of fluctuating constituent of momentum at the flow field points (henceforth - the moment-of-momentum) with $\boldsymbol{R}$ standing for the arm of the moment and $\Omega$ has the sense of the average angular velocity of rotation of the medium particles at the flow field points in respect to the random curvature centers of the velocity fluctuation streamlines passing these points. As the characteristics of a dynamical-kinematical state of the flow-field at the flow-field points the defined $\boldsymbol{M}$ and $\Omega$ determine the energy $K^{\Omega}=\frac{1}{2} \boldsymbol{M} \cdot \Omega$ specified as a part of the total turbulence energy $K=\frac{1}{2}\left\langle v^{\prime 2}\right\rangle$ represented as

$$
K=K^{\Omega}+K^{0},
$$

where $K^{0}=\frac{1}{2}\left\langle\boldsymbol{M}^{\prime} \cdot \Omega^{\prime}\right\rangle$ in which $\boldsymbol{M}^{\prime}=\boldsymbol{v}^{\prime} \times \boldsymbol{R}-\boldsymbol{M}$ and $\boldsymbol{\Omega}^{\prime}=\boldsymbol{v}^{\prime} \times \boldsymbol{k}-\boldsymbol{\Omega}$. The energy split in (3) shows the medium turbulence split into the orientated and the nonorientated constituents characterized by the pair $\boldsymbol{M}, \Omega$ and by $K^{0}$, respectively. Finally, proceeding from the sense of $\boldsymbol{M}$ and $\Omega$, it is natural to connect them by

$$
\boldsymbol{M}=\boldsymbol{\eta} \cdot \Omega,
$$

defining the tensor of effective moment of inertia $g$.

Let us emphasize the following:

1) The turbulence properties reflected by $\boldsymbol{M}$ and $\Omega$ relate the average turbulent continua to the class of MF with their MF properties reflecting the local effect of the prevailing orientation of eddy rotation;

2) Constituting $\boldsymbol{M}$ and $\boldsymbol{\Omega}$ identically vanishing, the CTM either confines its applicability to the situation with the correlations expressed by (1) and (2) absent (which is a physical assertion) or excludes axiomatically the curvature of the velocity fluctuation streamlines from the set of characteristics of the flow-field states in infinitesimal surroundings of the flow-field points;

3) The split of turbulence into the orientated and the non-orientated constituents reserves to the CTM the competence of describing the non-orientated constituent of turbulence;

4) The turbulence properties reflected by (1)-(3) provide a possibility to introduce the RK conception in an explicit form (in the two-scale approximation) to the average description of turbulence.

\subsection{The Balance Equations}

The following particularizes the explained in 2.1 setup of 
turbulence description in terms of differential balance equations for the average momentum, for the moment-ofmomentum, for different energy constituents and for the concentration of scalar substance.

\subsubsection{The Balance Equations for the Momentum and Moment-of-Momentum}

In the universal form the differential balance equations for the momentum and the moment-of-momentum write as [24]:

$$
\begin{gathered}
\rho \frac{\mathrm{d}}{\mathrm{d} t} \boldsymbol{u}=\left\{\sigma_{i j, j}\right\}+\rho \boldsymbol{F}, \\
\rho \frac{\mathrm{d}}{\mathrm{d} t} \boldsymbol{M}=\left\{m_{k j, j}\right\}-\boldsymbol{\sigma}+\rho \boldsymbol{m} .
\end{gathered}
$$

In (5) and (6): d/d $t=\partial / \partial t+\boldsymbol{u} \cdot \nabla ; \rho$ is the medium density; $\sigma_{i j}$ are the components of the stress tensor; $\boldsymbol{F}$ is the density (per unit mass) of the body force (henceforth - the body force); $m_{\mathrm{kj}}$ are the components of the moment stress tensor describing the diffusive transport of $\boldsymbol{M} ; \boldsymbol{\sigma}$ is the dual vector to the antisymmetric constituent of the stress tensor coupling the fields of momentum and the moment-of-momentum; $\boldsymbol{m}$ is the density (per unit mass) of the body moment (henceforth - the body moment) acting on the medium; the index after the subscript comma denotes differentiation along the respective space coordinate while the Einstein summation is assumed and the equivalent notation arbitrary tensor or vector quantity $\equiv\{$ components of this quantity $\}$ is applied.

The characterization of the turbulent flow-field explained in 2.1 specifies $\boldsymbol{u}$ and $\boldsymbol{M}$ in (5) and (6) as the average flow velocity and the moment-of-momentum defined in (1), respectively, while Equation (5) is the balance equation for the average momentum and Equation (6) is the equation obtained from the averaged difference of the balance equation of instantaneous momentum and Equation (5) vector-multiplied by $\boldsymbol{R}$ from the right. The derivation procedure provides all terms in (5) and (6) with specific expressions via the momentary flow-field characteristics [23]. In particular, it specifies the stress tensor as $\sigma_{i j}=\left\langle\sigma_{i j}^{m}\right\rangle+\sigma_{i j}^{t}$, where $\sigma_{i j}^{m}$ denote the components of the molecular stress tensor and $\sigma_{i j}^{t}=-\rho\left\langle v_{j}^{\prime} v_{i}^{\prime}\right\rangle$ denote the components of the turbulent stress tensor, and the body moment $\boldsymbol{m}$ as

$$
\boldsymbol{m}=\boldsymbol{m}_{1}+\boldsymbol{m}_{2}+\boldsymbol{m}_{f},
$$

where $\boldsymbol{m}_{1}=\left\langle\boldsymbol{v}^{\prime} \times \partial \boldsymbol{R} / \partial t\right\rangle, \quad \boldsymbol{m}_{2}=(\nabla \boldsymbol{u}) \cdot \boldsymbol{M}$ and

$$
\boldsymbol{m}_{f}=\frac{1}{\rho}\left\langle\boldsymbol{f}^{\prime} \times \boldsymbol{R}\right\rangle
$$

in which $f^{\prime}$ denotes the fluctuating constituent of the body force acting on the medium.

Notice, that the asserted by Equations (5) and (6) asymmetry of the turbulent stress tensor has the same origin as the non-triviality of the turbulent flow-field characteristics defined in (1) and (2).

\subsubsection{The Energy Balance Equations}

The full set of the energy balance equations of the PCTM comprise the balance equations for $K^{u}=\frac{1}{2} u^{2}$ (where $u=|\boldsymbol{u}|$ ) (derived as the scalar product of Equation (5) and $\boldsymbol{u}$ ), for $K^{\Omega}$ (deduced for $q=J \hat{\mathbf{1}}$ with $J=$ const, where $\hat{\mathbf{1}}$ denotes the unit tensor with the components $\delta_{i j}$, as the scalar product of Equation (6) and $\boldsymbol{\Omega}$ ), for $K^{0}$ (derived as the difference of equations of balance of turbulence energy $K$ and $K^{\Omega}$ ) and for internal (thermal) energy $U$, written as:

$$
\begin{gathered}
\rho \frac{\mathrm{d}}{\mathrm{d} t} K^{u}=\nabla \cdot \boldsymbol{h}^{u}-A-\Psi^{u}-\psi^{u}+\rho q^{u}, \\
\rho \frac{\mathrm{d}}{\mathrm{d} t} K^{\Omega}=\nabla \cdot \boldsymbol{h}^{\Omega}+A+B-\Psi^{\Omega}-\psi^{\Omega}+\rho q^{\Omega}, \\
\rho \frac{\mathrm{d}}{\mathrm{d} t} K^{0}=\nabla \cdot \boldsymbol{h}^{0}+\Psi^{u}+\Psi^{\Omega}-B-\psi^{0}+\rho q^{0}, \\
\rho \frac{\mathrm{d}}{\mathrm{d} t} U=\nabla \cdot \boldsymbol{h}^{U}+\psi
\end{gathered}
$$

In (8)-(11): $\boldsymbol{h}^{u}, \boldsymbol{h}^{\Omega}, \boldsymbol{h}^{0}, \boldsymbol{h}^{U}$ denote diffusive flux vectors of $K^{u}, K^{\Omega}, K^{0}$ and $U$, respectively; $A=-\sigma \cdot \omega$ (where $\omega=\frac{1}{2} \nabla \times \boldsymbol{u}$ is the vorticity) denotes the work realizing the energy exchange between $K^{u}$ and $K^{\Omega}$; $B=\rho \boldsymbol{m}_{2} \cdot \Omega$ denotes the work realizing the energy exchange between $K^{\Omega}, K^{0} ; \Psi^{u}=\sigma_{(j i)}^{t} u_{(i, j)}$ (in which $\sigma_{(i j)}^{t}=\frac{1}{2}\left(\sigma_{i j}^{t}+\sigma_{j i}^{t}\right)$ and $\left.u_{(i, j)}=\frac{1}{2}\left(u_{i, j}+u_{j, i}\right)\right)$ denotes the work realizing the energy $K^{u}$ scatter into the energy $K^{0}$; $\Psi^{\Omega}=m_{i j} \Omega_{i, j}+\sigma \cdot(\boldsymbol{\Omega}-\boldsymbol{\omega})-\rho \boldsymbol{m}_{1} \cdot \boldsymbol{\Omega}$ denotes the work resulting in the scatter of energy $K^{\Omega}$ into the energy $K^{0} ; \psi^{u}=\left\langle\sigma_{(i j)}^{m}\right\rangle u_{(i, j)} \geq 0, \psi^{\Omega} \geq 0$ and $\psi^{0} \geq 0$ reflect the molecular dissipation of the energies $K^{u}, K^{\Omega}$ and $K^{0}$, respectively; $\psi=\psi^{u}+\psi^{\Omega}+\psi^{0} ; \rho q^{u}=\rho \boldsymbol{F} \cdot \boldsymbol{u}$, $\rho q^{\Omega}=\rho \boldsymbol{m}_{f} \cdot \boldsymbol{\Omega}$ and $\rho q^{0}$ describe the effect of external fields on the energies $K^{u}, K^{\Omega}$ and $K^{0}$.

A substantial implication of the energy balance situation represented by (8)-(11) is the specification of the pairs of "generalized forces" and the respective "generalized velocities" as $\left(\sigma_{(i j)}^{t} u_{(i, j)}\right),\left(m_{i j}, \Omega_{i, j}\right),(\boldsymbol{\sigma}, \boldsymbol{\Omega}-\boldsymbol{\omega})$ and $\left(-\rho \boldsymbol{m}_{1}, \Omega\right)$. Each of the pairs determines an independent physical process realizing the scatter of the energies $K^{u}$ and/or $K^{\Omega}$ into the energy $K^{0}$. Notice that, unlike the positive $\Psi^{u}$ and $\Psi^{\Omega}$, the work $A$ and the work $B$ may be either positive or negative. In particular, positive $A$ is related to the energy $K^{\Omega}$ feeding on the energy $K^{u}$ while the negative $A$ declares the situation of the eddy-to-mean energy conversion accompanied with 
the up-gradient momentum transfer. For the stationary and homogeneous situation the latter possibility assumes $\rho q^{\Omega}$ positive, i.e. external fields feeding the energy $K^{\Omega}$. The other note relates to the energy $K^{0}$ feeding on the energies $K^{u}$ and $K^{\Omega}$, from which the RK conception excludes the first energy source and the CTM excludes the second. The concurrent inclusion of both situations in set (8)-(11) suggests the compilation of the RK conception (in two-scale approximation) as well as the CTM within one unique formalism.

\subsubsection{The Balance Equation for the Scalar Substance}

The turbulence properties reflected by the defined in (1) and (2) quantities introduce a change not only into the description setup of turbulent motions explained in 2.2.1 and 2.2.2 but also into the description of turbulent transport processes. Indeed, denoting by $c, C=\langle c\rangle$ and $c^{\prime}=c-C$ the instantaneous, average and fluctuating concentration of an arbitrary scalar substance (concentration of ingredients, temperature, etc.), respectively, and using the identity $\boldsymbol{v}^{\prime}=\boldsymbol{R} \times\left(\boldsymbol{v}^{\prime} \times \boldsymbol{R}\right) / R^{2}$, the turbulent flux vector of the substance, $\boldsymbol{h}_{\mathrm{C}}=-\left\langle\boldsymbol{v}^{\prime} c^{\prime}\right\rangle$, in the balance equation for $C$,

$$
\frac{\mathrm{d}}{\mathrm{d} t} C=\nabla \cdot \boldsymbol{h}_{\mathrm{C}}+Q,
$$

where $Q$ denotes the body-source of $C$, becomes represented as

$$
\boldsymbol{h}_{\mathrm{C}}=\boldsymbol{h}_{\mathrm{C}}^{\Omega}+\boldsymbol{h}_{\mathrm{C}}^{0},
$$

In (13) $\boldsymbol{h}_{C}^{0}=\left\langle\Omega^{\prime} \times c^{\prime} \boldsymbol{R}\right\rangle$ and $\boldsymbol{h}_{C}^{\Omega}=\boldsymbol{\Omega} \times\left\langle c^{\prime} \boldsymbol{R}\right\rangle$ describe the turbulent transport of $C$ by the non-orientated and by the orientated turbulence constituents, respectively.

\section{The RAT Theory}

\subsection{The Closure Assumptions}

The RAT theory [23] realizes the all-purpose formalism of the PCTM explained above within a specific solution of the closure problem of the balance Equations (5), (6) and (12), formulated in three steps. The first step (already applied while deriving Equation (9)) constitutes

$$
\eta=J \hat{\mathbf{1}}
$$

where $J>0$. Notice that $\sqrt{J}$ determines the characteristic length scale of eddies contributing to $\boldsymbol{M}$ and $\Omega$. Unlike $\boldsymbol{R}$, the length scale $\sqrt{J}$ is an average quantity.

In the second step the "generalized forces", revealed in the analysis of energy balance in 2.2.2, are set to linearly depend on the respective "generalized velocities", written as

$$
\sigma_{(i j)}^{t}=-p \delta_{i j}+2 \mu u_{(i, j)}
$$

$$
\begin{gathered}
m_{i j}=\vartheta_{0} \Omega_{k, k} \delta_{i j}+\vartheta_{1} \Omega_{i, j}+\vartheta_{2} \Omega_{j, i}, \\
\sigma=4 \gamma(\Omega-\omega),
\end{gathered}
$$

and

$$
-\rho \boldsymbol{m}_{1}=4 \kappa \Omega \text {. }
$$

In (15)-(18): $p$ is the pressure; $\mu>0$ is the coefficient of turbulence shear viscosity; $\vartheta_{0}, \vartheta_{1}, \vartheta_{2} \geq 0$, are the diffusion coefficients of $\boldsymbol{M} ; \gamma \geq 0$ is the coefficient of turbulence rotational viscosity characterizing the shear stresses in the relative rotation, i.e. for $\Omega \neq \omega ; \kappa \geq 0$ interprets as the coefficient of decay of $\boldsymbol{M}$ due to the cascading process. Relations (15)-(17) are similar to the respective relations within the MF theory whereas the relation (18) reflects a fundamental difference in the properties of turbulent media and micropolar fluids - when the turbulence structure requires incessant restoration then the MF theory considers the media having a fixed structure.

The third step constitutes $\boldsymbol{h}_{C}^{0}$ and $\left\langle c^{\prime} \boldsymbol{R}\right\rangle$, determining $\boldsymbol{h}_{C}$ in (13), expressed as $\boldsymbol{h}_{\mathrm{C}}^{0}=k_{0} \nabla C$ and $\left\langle c^{\prime} \boldsymbol{R}\right\rangle=k_{1} \nabla C \times \Omega-k_{2} \nabla C$ (with the latter derived as the dependence of $\left\langle c^{\prime} \boldsymbol{R}\right\rangle$ on $\boldsymbol{\Omega}$ and $\nabla C$ linear in respect to the both arguments and vanishing for $\nabla C=0$ ) resulting in

$$
\boldsymbol{h}_{C}=\boldsymbol{\kappa} \cdot \nabla C .
$$

In (19) $₹$ is the turbulent transport tensor represented as

$$
\boldsymbol{K}=\boldsymbol{K}^{s}+\boldsymbol{K}^{a s},
$$

in which $\boldsymbol{K}^{s}=k_{0} \hat{\mathbf{1}}+k_{1}\left(\Omega^{2} \hat{\mathbf{1}}-\Omega \Omega\right)$ specifies the symmetric and $x^{a s}=-k_{2} \varepsilon \cdot \Omega$ (where $\mathcal{E}$ is the LeviCivita tensor) - the antisymmetric constituent of $\pi$. Equations (19) and (20) explain $k_{0}$ and $k_{1}$ as the positive coefficients characterizing normal (down-gradient) turbulent diffusion of $C$ and $k_{2}$-as the coefficient characterizing the cross-gradient turbulent diffusion of $C$. For $C$ not contributing to the density field the sign of $k_{2}$ specifies as negative. Otherwise the sign of $k_{2}$ becomes depending on whether the $\nabla C$ constituent perpendicular to the gravity acceleration amplifies of depresses $\Omega$.

\subsection{The Equations of the RAT Theory}

The closure relations (14)-(19), turn the balance Equations (5), (6) and (12) (henceforth $J, \mu, \gamma, \kappa, \vartheta_{0}, \vartheta_{1}, \vartheta_{2}, k_{0}$, $k_{1}$ and $k_{2}$ are constituted to be constants) into the following set of equations to determine $\boldsymbol{u}, \boldsymbol{\Omega}$ and $C$

$$
\begin{aligned}
\rho \frac{\mathrm{d} \boldsymbol{u}}{\mathrm{d} t}= & -\nabla p+(\mu+\gamma) \Delta \boldsymbol{u}+2 \gamma \nabla \times \Omega+\rho \boldsymbol{F}, \\
\rho J \frac{\mathrm{d} \boldsymbol{\Omega}}{\mathrm{d} t}= & \left(\vartheta_{0}+\vartheta_{2}\right) \nabla \nabla \cdot \Omega+\vartheta_{1} \Delta \Omega-4 \gamma(\boldsymbol{\Omega}-\boldsymbol{\omega}) \\
& -4 \kappa \boldsymbol{\Omega}+\rho J(\nabla \boldsymbol{u}) \cdot \boldsymbol{\Omega}+\rho \boldsymbol{m}_{f},
\end{aligned}
$$




$$
\frac{\mathrm{d}}{\mathrm{d} t} C=\nabla \cdot(\text { ス } \nabla C)+Q,
$$

where $z$ is specified in (20).

Let us accompany Equations (21)-(23) with the following comments.

1) The closure assumptions resulting in (21) and (22) specify also all terms in the energy balance Equations (8)-(10) providing any solution of (21) and (22) with a rigid physical sense expressed in energetic terms. In particular, the closure relation (17) specifies the work $A$ in (9) and (10) as $A=-4 \gamma(\Omega-\omega) \omega$ which sets the problem of eddy-to-mean energy conversion into the dynamical context expressed by (21) and (22) avoiding the application of negative viscosity [25] or the idealization of the 2D turbulence.

2) Delegating the description of the orientated (relatively large scale) turbulence constituent to the equation of the moment-of-momentum, the formalism of the RAT theory proves closer to the theory of MF than to the CTM, which ignores this equation in its setup.

3) The constituted in the CTM statement of symmetry of the turbulent stress tensor reads in terms of Equations (21) and (22) as the condition $4 \gamma(\boldsymbol{\Omega}-\boldsymbol{\omega}) \equiv 0$ holding either for $\gamma=0$ or for $\Omega \equiv \omega$ which enlightens the ambivalence of physical interpretation of the CTM. In the first case, if $\boldsymbol{m}_{f}=\boldsymbol{F}=0$ and if $\boldsymbol{\Omega}$ is identically zero at an initial time instant, then it appears vanishing also for all following time instants. In the second case Equation (22) should reduce to the equation for vorticity following from Equation (21) which takes place if $\kappa=0$ and $\vartheta_{1}=\mu \mathrm{J}$.

4) Insofar as $\nabla \cdot\left(\boldsymbol{z}^{a s} \cdot \nabla C\right)=-\boldsymbol{s} \cdot \nabla C$, in which $\boldsymbol{s}=k_{2} \nabla \times \Omega$, Equation (23) can be rewritten also as

$$
\frac{\mathrm{d}}{\mathrm{d} t} C+\boldsymbol{s} \cdot \nabla C=\nabla \cdot\left(\boldsymbol{z}^{s} \cdot \nabla C\right)+Q,
$$

explaining the effect of the cross-gradient turbulent transport similar to the advection by the velocity field of incompressible fluid $(\nabla \cdot s \equiv 0)$.

\subsection{Turbulent Flows in Specific Conditions}

\subsubsection{Description of Turbulent Flows under the Influence of External Fields}

The effect of external fields on turbulence is accounted for in (21) and (22) through the terms $\rho \boldsymbol{F}$ and $\rho \boldsymbol{m}_{f}$. In the following the situation is particularized for two cases.

The first case is related to the flows of electrically conductive media under the influence of external magnetic field for small magnetic Reynolds number values, where [26]

$$
\rho \boldsymbol{F}=\sigma_{E}\left[\langle\boldsymbol{E}\rangle \times \boldsymbol{B}_{0}+\left(\boldsymbol{B}_{0} \boldsymbol{B}_{0}-B_{0}^{2} \hat{\mathbf{1}}\right) \cdot \boldsymbol{u}\right]
$$

$$
\rho \boldsymbol{m}_{f}=-\frac{J}{2}(1-\beta) \sigma_{\boldsymbol{E}}\left(\boldsymbol{B}_{0} \boldsymbol{B}_{0}+B_{0}^{2} \hat{\mathbf{1}}\right) \cdot \Omega
$$

in which $\boldsymbol{B}_{0}$ denotes the induction of the external magnetic field, $\sigma_{E}$ is the coefficient of electrical conductivity and $0 \leq \beta \leq 1$ denotes certain phenomenological characteristic of the medium electric properties. Expression (26) evinces the effect of external magnetic field resulting for small magnetic Reynolds number values in a suppression of $\Omega$. The situation changes for the medium and/or large magnetic number values when the magnetic field may prove acting as a source of energy of the orientated constituent of turbulence or the medium turbulence may prove acting as a course of generation of magnetic field.

The second case is related to the flows under the influence of gravity force, where within the Boussinesq approximation [27] we have [26]

$$
\rho \boldsymbol{F}=\tilde{\rho} \boldsymbol{g}
$$

and

$$
\begin{aligned}
\rho \boldsymbol{m}_{f}= & -k_{1}[(\nabla \tilde{\rho} \cdot \boldsymbol{g}) \hat{\mathbf{1}}-(\nabla \tilde{\rho}) \boldsymbol{g}] \cdot \Omega \\
& -k_{2} \boldsymbol{g} \times \nabla \tilde{\rho},
\end{aligned}
$$

in which $\rho$ is the characteristic constant density of medium and $\tilde{\rho}$ is the medium actual average density, while $\tilde{\rho} \approx \rho$. Here, the integration of Equations (21) and (22) requires a specification of the equation of state expressing $\tilde{\rho}$ through the characteristics of medium ingredients contributing to $\tilde{\rho}$ and Equations (21) and (22) should be integrated together with the equations formulated for all medium ingredients contributing to $\tilde{\rho}$. Notice, that expression (28) compiles in one single formula the depression of the component of $\Omega$ perpendicular to the gravity acceleration caused by the stable stratification as well as its generation by the unstable stratification, which substantially simplifies the description of gravitation-related processes involving the both situations.

\subsubsection{Description of Turbulent Flows in Rotating Frames}

For the description of motion in a frame rotating with a constant angular velocity $\boldsymbol{\omega}^{0}$, the flow velocity $\boldsymbol{u}$ is replaced by $\boldsymbol{u}+\boldsymbol{\omega}^{0} \times \boldsymbol{r}$, where $\boldsymbol{r}$ denotes a radius-vector from the arbitrary point on the rotation axes to a point of the flow-field, $\omega$ and $\Omega$ are replaced by $\omega+\omega^{0}$ and $\boldsymbol{\Omega}+\boldsymbol{\omega}^{0}$, and $\mathrm{d} \boldsymbol{u} / \mathrm{d} t, \mathrm{~d} \boldsymbol{M} / \mathrm{d} t$ are replaced by $\mathrm{d} \boldsymbol{u} / \mathrm{d} t+\boldsymbol{\omega}^{0} \times \boldsymbol{u}, \quad \mathrm{d} \boldsymbol{M} / \mathrm{d} t+\boldsymbol{\omega}^{0} \times \boldsymbol{M}$. The changes result in the complementation of the right sides of (21) and (22), respectively, by the Coriolis force term

$$
\rho \boldsymbol{F}_{C}=2 \rho \boldsymbol{u} \times \boldsymbol{\omega}^{0}
$$

and by the additional body moment term 


$$
\rho \boldsymbol{m}^{0}=-4 \kappa \boldsymbol{\omega}^{0}+J \rho(\nabla \boldsymbol{u}) \cdot \boldsymbol{\omega}^{0}+\rho J \boldsymbol{\Omega} \times \boldsymbol{\omega}^{0}
$$

and in the replacement of the expression for $z^{s}$ by

$$
\boldsymbol{Z}^{s}=k_{0} \hat{\mathbf{1}}+k_{1}\left[\left|\boldsymbol{\Omega}+\boldsymbol{\omega}^{0}\right|^{2} \hat{\mathbf{1}}-\left(\boldsymbol{\Omega}+\boldsymbol{\omega}^{0}\right)\left(\boldsymbol{\Omega}+\boldsymbol{\omega}^{0}\right)\right] .
$$

The expressions (29)-(31) evidence about a substantial difference between the turbulence properties in the rotating and the non-rotating frames. So, the first term on the right side of (30) evinces the frame rotation preferring the anticyclonic (directed opposite to the direction of $\omega^{0}$ ) orientation of $\Omega$ (gyration effect). The work done by the moment $\rho \boldsymbol{m}^{0}$ may serve also as an additional cause of eddy-to-mean energy conversion etc.

\section{Examples of Application of the RAT Theory}

\subsection{One-Dimensional Flows in Plain Channels, Round Tubes, between Rotating Concentric Cylinders and Boundary Layers}

For one-dimensional flows in plain channels, round tubes, between rotating concentric cylinders [23], and in boundary layers [28] the Equations (21) and (22) simplify to

$$
\begin{aligned}
& \rho \frac{\partial \boldsymbol{u}}{\partial t}=-\nabla p+(\mu+\gamma) \Delta \boldsymbol{u}+2 \gamma \nabla \times \boldsymbol{\Omega}+\rho \boldsymbol{F}, \\
& \rho J \frac{\partial \boldsymbol{\Omega}}{\partial t}=\vartheta_{1} \Delta \boldsymbol{\Omega}-4 \gamma(\boldsymbol{\Omega}-\boldsymbol{\omega})-4 \kappa \boldsymbol{\Omega}+\rho \boldsymbol{m}_{f}
\end{aligned}
$$

where $\Omega$ and $\boldsymbol{u}$ are orientated perpendicularly and depend on the coordinate perpendicular to $\Omega$ and $\boldsymbol{u}$.

For $\boldsymbol{F}=\boldsymbol{m}_{f}=0$ the predicted by (32) and (33) velocity profiles were compared in [23] (for steady flows in plain channels, in round tubes and between rotating concentric cylinders) with data in [29-31] and (in case of oscillating flow in round tube) with data in [32]. For oscillating boundary layer generated by undulating free flow the predicted by (32) and (33) velocity profiles were compared in [28] with data in [33]. The predicted by (32) and (33) velocity profiles for $\boldsymbol{F}$ and $\boldsymbol{m}_{f}$ specified in (25) and (26) were compared with data in [34]. In all cases the predicted velocity profiles prove excellently matching the actual velocity data. Notice, that for $\kappa=0$ Equations (32) and (33) coincide in written form with the respective equations of the MF theory. However, the situations with $\kappa=0$ and $\kappa \neq 0$ prove reflecting physically different situations. In particular, for steady flows in round tubes and plain channels the solution of (32) and (33) predicts the flow velocity determined in the central part of the flow region by the effective viscosity $\mu_{\mathrm{ef}}=\mu+\gamma \kappa(\gamma+\kappa)$ which hints to a substantial role of the turbulence properties characterized by $\gamma$ and $\kappa$ in this region with the property characterized by $\mu$ play- ing (in harmony with the RK conception) a marginal role. In the central part of the flow, for $\kappa=0$, the turbulence properties appear represented by the turbulence shear viscosity only.

\subsection{Vertical Distribution of Concentration of Suspended Sediments in a River Estuary}

In [35] Equations (21)-(23) were applied to describe the vertical distribution of concentration of the suspended matter $(C)$ in a river estuary modeled as an open channel with the fixed bottom slope angle $(\alpha)$ and the timevarying free surface angle $(\beta=\beta(t))$. Restricting the consideration with $\alpha, \beta \ll 1$, the quasi-stationary flow regime and with the concentrations small enough to not influence the density field, Equations (21)-(23) read as

$$
\begin{gathered}
(\mu+\gamma) \frac{\partial^{2} u}{\partial z^{2}}-2 \gamma \frac{\partial \Omega}{\partial z}+\rho g(\alpha+\beta(t))=0, \\
\vartheta_{1} \frac{\partial^{2} \Omega}{\partial z^{2}}-4(\gamma+\kappa) \Omega+2 \gamma \frac{\partial u}{\partial z}=0, \\
\frac{\partial}{\partial z}\left[\left(k_{0}+k_{1} \Omega^{2}\right) \frac{\partial C}{\partial z}\right]+Q=0,
\end{gathered}
$$

where $Q=w \partial C / \partial z$, in which $w$ is the settling velocity and $z$ is the vertical coordinate directed upward. The term $\rho g(\alpha+\beta)$ in (34) expresses the summary effect of the along-flow pressure gradient and of the gravity force. The determined from (34)-(36) vertical distributions of $C$ were compared with concentration of the resuspended sediments observed in the Jiaojiang Estuary (China) [36] for different time instants of a spring tide cycle. The comparison showed that the derived analytical formula for $C$ embraces two observed basic types of vertical distribution of concentration, one with a monotonic decrease of concentration gradient with distance from the bottom and the other with a gradient maximum (lutocline) located at some distance from the bottom. (The both types of vertical distribution of suspended sediments were detected also in the bottom layer of natural water body, studied in [37]).

\subsection{Vertical Structure of the Upper Ocean}

Consider now the situation in the upper ocean in Boussinesq approximation specified by $\boldsymbol{u}=\left(u_{x}(z), u_{y}(z), 0\right)$, $\Omega=\left(\Omega_{x}(z), \Omega_{y}(z), 0\right)$ and $|\Omega| \gg\left|\omega^{0}\right|$ (the right-hand Cartesian coordinate system $(x, y, z)$ with $z \geq 0$ directed downward is assumed; hereafter $\omega^{0}$ is the angular velocity of the Earth rotation). From Equations (21)(23), where $\rho \boldsymbol{F}$ is determined according to (27) and (29) as $\tilde{\rho} \boldsymbol{g}+2 \rho \boldsymbol{u} \times \boldsymbol{\omega}^{0}, \quad \rho \boldsymbol{m}_{f}$ is determined according to (28) as $\rho \boldsymbol{m}_{f}=-k_{1} g(\partial \tilde{\rho} / \partial \mathrm{z}) \boldsymbol{\Omega}$ and with $Q=0$ we have 


$$
\begin{gathered}
\rho \frac{\partial \boldsymbol{u}}{\partial t}=-\nabla p+(\mu+\gamma) \frac{\partial^{2}}{\partial z^{2}} \boldsymbol{u}+2 \gamma \nabla \times \boldsymbol{\Omega}+2 \rho \boldsymbol{u} \times \boldsymbol{\omega}^{0} \\
\rho J \frac{\partial \boldsymbol{\Omega}}{\partial t}=\vartheta_{1} \frac{\partial^{2}}{\partial z^{2}} \boldsymbol{\Omega}-4 \gamma(\boldsymbol{\Omega}-\boldsymbol{\omega})-4 \kappa \boldsymbol{\Omega}-k_{1} g \frac{\partial \tilde{\rho}}{\partial z} \boldsymbol{\Omega} \\
\nabla p=\tilde{\rho} g \\
\frac{\partial \tilde{\rho}}{\partial t}=\frac{\partial}{\partial z}\left[\left(k_{0}+k_{1} \Omega^{2}\right) \frac{\partial \tilde{\rho}}{\partial z}\right]
\end{gathered}
$$

Equations (37)-(39) explain the stable stratification in supressing the $\Omega$ constituent perpendicular to the gravity acceleration together with an increase of vertical gradient of density, and the unstable stratification in amplifying the constituent perpendicular to the gravity acceleration together with a decrease of vertical gradient of density.

For the constant $\tilde{\rho}$ the solution of (37) and (38) [38, $39]$ for the velocity sums up from two addends reflecting the Stokes drift effect [40] and the classical Ekman vertical velocity profile [27] with the turbulence viscosity replaced by the effective viscosity $\mu_{\text {ef }}$. The solution explains the Stokes drift effect in good agreement with data in [41], in diminishing the angle between the flow velocity and the shear stress. To demonstrate the stratification effect Equations (37) and (38) were solved in [39] for $\tilde{\rho}$ constant everywhere instead of a density jump at a certain depth modeling the assumed location of thermocline in summer and winter. The calculated vertical distribution of velocity was compared in both cases with the velocity data in [42] showing a good agreement in the dominating quality.

In $[43,44]$, Equations (37)-(39) were applied to model the reaction of the upper ocean to periodical cooling and heating. Here $\partial \tilde{\rho} / \partial z=-\alpha \partial T / \partial z+\beta \partial S / \partial z$, where $T$ is temperature, $S$ is salinity, $\alpha$ is the coefficient of thermal expansion and $\beta$ characterizes the salinity contraction variance. It is shown that Equations (37)-(39) predict the formation of a typical for the upper ocean vertical density profile with the relatively uniform density distribution in the layer next to the ocean surface separated from lower layers by a stratum of relatively abrupt density jump in a reasonable agreement with the observed data.

\subsection{Conjoint Effect of the Baroclinic Instability and the Rotational Viscosity of Turbulence}

In $[45,46]$, Equations (21), together with $\rho \boldsymbol{F}$ replaced by $\rho \boldsymbol{F}_{C}$ in (29), and (22), with $\rho \boldsymbol{m}_{f}$ specified in (28), were applied to calculate ageostrophic correction $u^{\text {ag }}$ to geostrophically predicted net transport of the Antarctic Circumpolar Current [47] from the observed spatial distribution of $\tilde{\rho}$. The idea lies in the determination of a correction from the balance condition of vertical con- stituent of the Coriolis force with the vertical constituent of the force described by the term $2 \gamma \nabla \times \Omega$ in (21), giving

$$
u^{\mathrm{ag}}=\frac{\gamma}{\rho r \omega^{0} \cos ^{2} \vartheta} \frac{\partial}{\partial \vartheta}\left(\Omega_{\phi} \cos \vartheta\right),
$$

where $\Omega_{\phi}$ is determined from the zonal projection of (22) as

$$
\Omega_{\phi}=-k_{2} g \frac{1}{r} \frac{\partial \tilde{\rho}}{\partial \vartheta}\left[4(\gamma+\kappa)+k_{1} g \frac{\partial \tilde{\rho}}{\partial z}\right]^{-1} .
$$

Equations (40) and (41) explain $u^{\mathrm{ag}}$ formed as a conjoint effect of the rotational viscosity of medium turbulence $(\gamma)$ and the baroclinic instability (characterized by $\left.k_{2} \geq 0\right)$. The both mentioned effects are excluded within the CTM. A similar setup was applied in [48] to explain the formation of zonal winds in planetary atmosphere with the main interest to the formation of easterlies in the equatorial zone showing a reasonable agreement with the observed velocity data in [49].

\subsection{Gyration Effect}

In sub-subsection 3.3.2 it was pointed out that the frame rotation prefers the anticyclonic orientation of $\Omega$. The represented in [50,51] zonally averaged $\Omega=(0,0, \Omega)$ and $\boldsymbol{M}=(0,0, M)$ estimated from the global surface drifter data sets [52] (the right-hand coordinate system $(\vartheta, \varphi, z)$, where $-\frac{\pi}{2} \leq \vartheta \leq \frac{\pi}{2}$ is latitude, $\varphi$ is longitude and axis $z$ of the coordinate system is directed upward is applied) confirm this conclusion, called in [50,51] the gyration effect. In the following some theoretical aspects of the gyration effect from the position of the RAT theory are commented.

\subsubsection{Theoretical Evidence of the Gyration Effect}

First consider how the gyration effect agrees with Equations (22) and (30). Restricting the consideration with the effects of diffusion of moment-of-momentum neglected we have from (22) and (30)

$$
\begin{aligned}
& \rho J \boldsymbol{u} \cdot \nabla_{h}\left(\Omega+\omega^{0} \sin \vartheta\right) \\
& =-4 \gamma(\Omega-\omega)-4 \kappa\left(\Omega+\omega^{0} \sin \vartheta\right) \\
& +\rho J \frac{\partial u_{z}}{\partial z}\left(\Omega+\omega^{0} \sin \vartheta\right)
\end{aligned}
$$

where $\nabla_{h}$ denotes horizontal gradient operator. Insofar as from the continuity equation $\nabla \cdot \boldsymbol{u}=0$ follows that $\nabla_{h} \cdot \boldsymbol{u}=-\partial u_{z} / \partial z \quad$ Equation (42) rewrites also as

$$
\begin{aligned}
& \rho J \nabla_{h} \cdot \boldsymbol{u}\left(\Omega+\omega^{0} \sin \vartheta\right) \\
& =-4 \gamma(\Omega-\omega)-4 \kappa\left(\Omega+\omega^{0} \sin \vartheta\right)
\end{aligned}
$$

For $\boldsymbol{u}=0$ Equation (43) gives 


$$
\Omega=-\frac{\kappa}{\gamma+\kappa} \omega^{0} \sin \vartheta .
$$

Expression (44) explains the gyration effect generated by the rotation of frame in balance of the shear in relative rotation and of the decrease of a prevailing orientation of eddy rotation in cascading process.

\subsubsection{Anomalous Turbulent Diffusive Transport}

Paper [53] exploits (44) within a model explaining the observed tongue-like structure of the salinity distribution in the region of the Gibraltar Salinity Anomaly (GSA) [54]. The tongue-like structure of the anomaly is explained as a result of cross-gradient turbulent diffusion. This explanation follows from Equation (24) for $C$ specified as salinity $(S)$ depending on $\varphi$ (longitude) and $\vartheta$ (latitude) only, written as

$$
\boldsymbol{s} \cdot \nabla S=\nabla \cdot\left[\left(k_{0}+b \sin ^{2} \vartheta\right) \nabla S\right],
$$

where $\boldsymbol{s}=a \cos \vartheta \boldsymbol{e}_{\phi}$ (in which $\boldsymbol{e}_{\phi}$ is the unit vector directed to the east, $\left.a=k_{2} \gamma \omega^{0} /(\gamma+\kappa)\right)$ and $b=k_{1}\left(\gamma \omega^{0} /(\gamma+\kappa)\right)^{2}$. According to Equation (45) the gyration effect stretches the salinity distribution out in the east-west direction $\left(k_{2}<0\right)$ and shifts the maxima of the latitudinal distribution of salinity increasingly to the south with distance from the Gibraltar Strait in good qualitative agreement with the observed situation represented in [54].

\subsubsection{Eddy-to-Mean Energy Transfer in Geophysical} Jet Flows

For $\boldsymbol{u} \neq 0$ and for $\Omega+\omega^{0} \sin \vartheta$ conserved in a flow $\left(\nabla_{h} \cdot \boldsymbol{u}\left(\Omega+\omega^{0} \sin \vartheta\right)=0\right)$ from (42) we have

$$
\Omega=-\frac{\kappa}{\gamma+\kappa} \omega^{0} \sin \vartheta+\frac{\gamma}{\gamma+\kappa} \omega .
$$

Using (46), the work $A=-\sigma \cdot \omega$ performing the energy exchange between the average flow and the orientated turbulence constituent, expresses as

$$
A=4 \frac{\gamma \kappa}{\gamma+\kappa}\left(\omega+\omega^{0} \sin \vartheta\right) \omega
$$

Paper [55] employs (46) and (47) explaining the upgradient momentum transfer and eddy-to-mean energy conversion $(A<0)$ avoiding the negative viscosity problem [25] or the application of the conception of $2 \mathrm{D}$ turbulence [56,57]. The situation was particularized for the cross-sections of Gulf Stream at $26^{\circ} \mathrm{N}$ (Florida Straits) and at $35^{\circ} \mathrm{N}$ (Onslow Bay) where, in harmony with (47) and the observational data in [58], the regions with the up-gradient momentum transfer and eddy-tomean energy conversion were observed at the anticyclonic sides $(\omega<0)$ of the stream.

As an additional result, the discussion evidences about the insufficiency of the velocity covariance data for unambiguous solution of the problem of turbulent stress tensor properties. In particular, for antisymmetric stresses the velocity covariance determines the stress tensor components with the accuracy up to the sign, specified from consideration of balance of internal moments acting in the medium.

\subsubsection{Topographically Generated Flows}

Using the continuity equation in a shallow water region, written as $\partial u_{z} / \partial z=H^{-1} \boldsymbol{u} \cdot \nabla H$ [27], from Equation (42) it follows, that

$$
\rho J \mathbf{u} \cdot \nabla \frac{\Omega+\omega^{0} \sin \vartheta}{H}=-4 \gamma \frac{\Omega-\omega}{H}-4 \kappa \frac{\Omega+\omega^{0} \sin \vartheta}{H} .
$$

For $\left(\Omega+\omega^{0} \sin \vartheta\right) / H$ conserved in a shallow water region we have

$$
\Omega=-\omega^{0} \sin \vartheta+C H,
$$

where $C$ is constant, and

$$
\omega=-\omega^{0} \sin \vartheta+\frac{\gamma+\kappa}{\gamma} C H .
$$

Expressing $C$ through the depth $H_{\text {cr }}$, where the energy $K^{\Omega}$ scatter into energy $K^{0}$ obtains its minimum, gives [59]

$$
C=\frac{\gamma}{\gamma+\kappa} \frac{1}{H_{\mathrm{cr}}} \omega^{0} \sin \vartheta
$$

and from (49) and (50) we have

$$
\Omega=-\left(1-\frac{\gamma}{\gamma+\kappa} \frac{H}{H_{\mathrm{cr}}}\right) \omega^{0} \sin \vartheta
$$

and

$$
\omega=-\left(1-\frac{H}{H_{\mathrm{cr}}}\right) \omega^{0} \sin \vartheta .
$$

Equations (51) and (52) agree with (44) for $H=H_{\text {cr }}$ suggesting identification of the actual depth $H$ with $H_{\text {cr }}$ in the open part of the water-body. In the alongshore region (52) declares that $\omega \neq 0$ evidencing about a nonvanishing velocity of flow in this region. In particular, in the alongshore region of a closed water-body the generated flow velocity is directed anticyclonically and around islands cyclonically. For $\kappa=0 \quad$ Equations (51) and (52) reduce to the condition of conservation of the potential vorticity predicting the motion in regions with sloped bottom, though leaving open the question about the source of motion energy. Unlike the discussions in [6062] suggesting to overcome this shortcoming within rather sophisticated theoretical constructions, the mechanism suggested above explains the energy supply from the energy associated with the gyration effect converted to the flow energy in shallow water regions. 


\subsection{Summary to the Commented Applications of the RAT Theory}

According to the described above applications, the RAT theory unites a broadened physical background of its setup with a noteworthy simplification of discussion of turbulence-related problems. The simplification follows from the split of turbulence into the orientated (relatively large scale) and the nonorientated (relatively small scale) constituents with the orientated constituent of turbulence dominating in the formation of properties of average flow. Contrary to the RAT theory, the CTM unites a simplification of its setup by neglecting the orientated turbulence constituent with complications in its applications following from the inconsistency between the absence of the orientated constituent of turbulence in the CTM setup and its presence in actual flows.

Despite focusing on the orientated turbulence constituent, the commented applications demonstrate considerable perspectives of the RAT theory. So, the examples show the ability of the RAT theory to describe the eddy-to-mean energy conversion avoiding the negative viscosity or evading the actual 3D structure of turbulence. The examples demonstrate also substantial perspectives of the RAT theory proceeding from the particularization of the interaction of turbulent flows with external fields and from the distinguishing the turbulence properties in rotating and non-rotating frames. The latter actualizes within the available ocean surface drifter data providing a plain observational evidence to the prevailing anticyclonic orientation of eddy rotation (gyration effect) which has been excluded within the CTM. Due to the inclusion of the gyration effect in its setup, the RAT theory sets this effect into the dynamic context required to explain the physical causes of the effect and its impact to the dynamical, energetic and to some other (like transport) processes in the upper ocean.

Despite the transparency of the applications formulated as a direct inference from the same set of equations, the focus of the applications on the orientated turbulence constituent turns the formulated results incompatible with the respective results formulated on the bases of the CTM. The indicated discrepancy increases due to the following from the RAT theory deficiency of contemporary methods of experimental research of turbulence adjusted to the requirements of the CTM.

\section{Conclusions}

The PCTM is an implication of the physical-historical point of view to the turbulence problem summarized as the PDT [22]. It advances the TM realizing a small but effective in its outcome modification at the very origin of the setup of the TM. The modification stands in complementation of characterization of the flow states in infinitesimal surroundings of flow points besides the flow velocity at these points also by the curvature of the velocity fluctuation streamlines passing these points. The introduced modification was aimed to clarify the classical conflict between the CTM and the RK conception actualized by the idea about applicability of the theory of MF to the description of turbulent flows. The PCTM accomplishes the task by compiling the CTM and the RK conception in a single theoretical construction. The RAT theory (complementing the universal formalism of the PCTM by the appropriate closure assumptions) justifies the applied modification from the pragmatic point of view. It compiles a substantial enlargement of the competence of the TM with a considerable simplification of the discussion without losing the physical rigidity. Besides grounding the RAT theory, the PCTM (especially if complemented in its setup with the method of decomposition of turbulent flow fields discussed and applied in [19-21]) makes the turbulence problem an interesting subject for theoretical discussions. As a mechanical outcome of the PDT the PCTM esteems also the PDT as a whole.

Unlike the PDT, the dominating up-to-date look on the turbulence problem reduces it to a huge number of particular problems stressing rather on their particularities than on their commonness, relates the RK conception to the ideas of the past not worthy to be revived in modern time and believes the fundamental aspects of the turbulence problem belonging rather to mathematics than to physics. The conflict emerged between the CTM and the PCTM is enforced by the criticism of the PCTM in address of the CTM. The conflict has all aspects archetypal to the paradigmatic conflicts in science, always accompanied with the critics of the old paradigm from the point of view of the novel paradigm and avoiding the discussions which may insinuate doubts about the grounding statements of the old paradigm.

In the end, any paradigmatic change in the science is always preceded by the superfluous aplomb of the former paradigm in its consummation, loss of adeptness for self-criticism and relating the unresolved problems to the solution nuances which cannot attaint the existing paradigm as a whole. Though, the unresolved nuances may incidentally actualize. Not finding answers within the dominating paradigm they start looking for answers on a wider scientific background embracing also neighboring science fields. If succeeding, the new expanded point of view may develop into an independent paradigm clarifying its relation with the former paradigm through a paradigmatic conflict. This is just the situation with the formulation of the RAT theory, the PCTM and the PDT. Started with formulation of the RAT theory initiated by the discussed in 70-es idea about applicability of the theory of MF to the description of turbulent flows, the for- 
mulation of this theory actualizes the RK conception as well as evinces the incompatibility of the RK conception with the CTM. The incompatibility raises several questions and the need to look for answers to these questions within the frames of the general principles of statistical physics collected together as the PDT. It motivates also the formulation of the PCTM within the classical formalism with an axiomatic change in the setup. This kind of the setup turns the physical background of the PCTM absolutely transparent and mandates the opponents either to agree with the suggested change or to reject the change by applying physical arguments. The fact that neither of the possibilities has realized characterizes the emerged paradigmatic conflict so deep that usually characterizes breaking points in the respective science fields.

\section{Acknowledgements}

The preparation of the paper was supported by grant ETF9381 of the Estonian Science Foundation. The author thanks Dr. Aleksander Toompuu for the idea to determine the gyration effect from the ocean surface drifter data providing the grounding statements of the RAT theory with observational evidence.

\section{REFERENCES}

[1] L. F. Richardson, "Weather Prediction by Numerical Process," Cambridge University Press, Cambridge, 1922.

[2] A. N. Kolmogorov, "The Local Structure of Turbulence in Incompressible Viscous Fluids for Very Large Reynolds Numbers," Doklady Akademii Nauk SSSR, Vol. 30, 1941, pp. 376-387 (in Russian).

[3] A. C. Eringen, "Microcontinuum Field Theories II. Fluent Media,” Krieger Pub. Co., Malabar, 1980.

[4] J. S. Dahler and L. F. Scriven, "Angular Momentum of Continua," Nature, Vol. 192, No. 4797, 1961, pp. 36-37. doi:10.1038/192036a0

[5] J. S. Dahler, "Transport Phenomena in a Fluid Composed of Diatomic Molecules," Journal of Chemical Physics, Vol. 30, No. 6, 1959, pp. 1447-1475.

doi:10.1063/1.1730220

[6] A. C. Eringen, "Theory of Micropolar Fluids," Journal of Mathematics and Mechanics, Vol. 16, No. 1, 1966, pp. 118.

[7] T. Ariman, M. A. Turk and D. O. Silvester, "Microcontinuum Fluid Mechanics-A Review," International Journal of Engineering Science, Vol. 11, No. 8, 1973, pp. 905-930. doi:10.1016/0020-7225(73)90038-4

[8] A. C. Eringen and T. S. Chang, "Micropolar Description of Hydrodynamic Turbulence," Advances in Materials Science and Engineering, Vol. 5, No. 1, 1970, pp. 1-8.

[9] A. C. Eringen, "Micromorphic Description of Turbulent Channel Flow," Journal of Mathematical Analysis and Applications, Vol. 39, No. 1, 1972, pp. 253-266. doi:10.1016/0022-247X(72)90239-9
[10] J. Peddieson, "An Application of the Micropolar Fluid Model to Calculation of Turbulent Shear Flow," International Journal of Engineering Science, Vol. 10, No. 1, 1972, pp. 23-32. doi:10.1016/0020-7225(72)90072-9

[11] V. N. Nikolajevskii, "Asymmetric Mechanics and the Theory of Turbulence," Archiwum Mechaniki Stosowanej, Vol. 24, 1972, pp. 43-51.

[12] V. N. Nikolajevskiy, "Angular Momentum in Geophysical Turbulence: Continuum Spatial Averaging Method," Kluwer, Dordrecht, 2003.

[13] L. D. Landau and E. M. Lifshitz, "Statistical Physics," Pergamon Press, Oxford, 1980.

[14] A. Ishiara, "Statistical Physics," Academic Press, New York-London, 1971.

[15] J. Heinloo, "Phenomenological Mechanics of Turbulent Flows," Valgus, Tallinn, 1984 (in Russian).

[16] J. Heinloo, “Turbulence Mechanics," Estonian Academy of Sciences, Tallinn, 1999 (in Russian).

[17] J. Heinloo, "On Description of Stochastic Systems," Proceedings of the Estonian Academy of Sciences, Physics and Mathematics, Vol. 53, No. 3, 2004, pp. 186-200.

[18] J. Heinloo, "A Setup of Systemic Description of Fluids Motion," Proceedings of the Estonian Academy of Sciences, Vol. 58, No. 3, 2009, pp. 184-189. doi:10.3176/proc.2009.3.05

[19] J. Heinloo, "The Structure of Average Turbulent Flow Field," Central European Journal of Physics, Vol. 8. No. 1, 2010, pp. 17-24. doi:10.2478/s11534-009-0015-y

[20] J. Heinloo, "Setup of Turbulence Mechanics Accounted for a Preferred Orientation of Eddy Rotation," Concepts of Physics, Vol. 5, No. 2, 2008, pp. 205-219. doi:10.2478/v10005-007-0033-8

[21] J. Heinloo, "A Generalized Setup of the Turbulence Description," Advanced Studies in Theoretical Physics, Vol. 5, No. 10, 2011, pp. 477-483.

[22] J. Heinloo, "Physical Doctrine of Turbulence-A Review," International Journal of Research and Reviews in Applied Sciences, Vol. 12, No. 2, 2012, pp. 214-221.

[23] J. Heinloo, "Formulation of Turbulence Mechanics," Physical Review E, Vol. 69, No. 5, 2004, Article ID: 056317. doi:10.1103/PhysRevE.69.056317

[24] L. I. Sedov, “A Course in Continuum Mechanics," Wolters-Noordhoff, Groningen, 1971.

[25] V. P. Starr, "Physics of Negative Viscosity Phenomena," McGraw-Hill, New York, 1968.

[26] J. Heinloo, "The Description of Externally Influenced Turbulence Accounting for a Preferred Orientation of Eddy Rotation," European Physical Journal B, Vol. 62, No. 4, 2008, pp. 471-476. doi:10.1140/epjb/e2008-00187-8

[27] J. Pedlosky, “Geophysical Fluid Dynamics," Springer, New York, 1987.

[28] J. Heinloo and A. Toompuu, "A Model of Average Velocity in Oscillating Turbulent Boundary Layers," Journal of Hydraulic Research, Vol. 45, No. 5, 2009, pp. 676680. doi:10.3826/ihr.2009.3579

[29] J. Nikuradse, "Gesetzmässigkeiten der Turbulenten Strö- 
mung in Glatten Rohren," VDI-Forschungsheft No 356, 1932, pp. 1-36.

[30] G. Comte-Bellot and A. Craya, "Écoulement Turbulent entre Deux Parois Parallèles," Fiche Détaillée, Paris, 1965.

[31] V. N. Zmeikov and B. P. Ustremenko, "Study of Energetic and Heat Transfer in Round Channel with Inner Rotating Cylinder," Problems of Thermoenergetics and Applied Thermophysics-1, Academy of Science of Kazakhstan SSR, 1964, p. 153 (in Russian).

[32] V. I. Bukreejev and V. M. Shakhin, "Experimental Study of Unsteady Turbulent Flow in Round Tube," Aeromehanika, Nauka, Moscow, 1976, p. 180 (in Russian).

[33] I. G. Jonsson and N. A. Carlsen, "Experimental and Theoretical Investigations in an Oscillatory Turbulent Boundary Layer," Journal of Hydraulic Research, Vol. 14, No. 1, 1976, pp. 45-60. doi:10.1080/00221687609499687

[34] G. G. Branover and A. B. Tsinober, "Magnetic Hydromechanics of Incompressible Fluids," Nauka, Moscow, 1970 (in Russian).

[35] J. Heinloo and A. Toompuu, "A Model of Vertical Distribution of Suspended Matter in an Open Channel Flow," Environmental Fluid Mechanics, Vol. 11, No. 3, 2011, pp. 319-328. doi:10.1007/s10652-010-9180-1

[36] J. Jiang and A. J. Mehta, "Lutocline Behavior in High-Concentration Estuary," Journal of Waterway, Port, Coastal, and Ocean Engineering, Vol. 126, No. 6, 2000, pp. 324328. doi:10.1061/(ASCE)0733-950X(2000)126:6(324)

[37] J. Heinloo and A. Toompuu, "A Model of the Vertical Distribution of Suspended Sediments in the Bottom Layer of Natural Water Body," Estonian Journal of Earth Sciences, Vol. 59, No. 3, 2010, pp. 238-245. doi:10.3176/earth.2010.3.05

[38] J. Heinloo and A. Toompuu, "A Modified Ekman Layer Model," Estonian Journal of Earth Sciences, Vol. 60, No. 2, 2011, pp. 123-129. doi:10.3176/earth.2011.2.06

[39] J. Heinloo and A. Toompuu, "A Modification of the Classical Ekman Model Accounting for the Stokes Drift and Stratification Effects," Environmental Fluid Mechanics, Vol. 12, No. 2, 2011, pp. 101-113. doi:10.1007/s10652-011-9212-5

[40] O. M. Phillips, "Dynamics of Upper Ocean," Cambridge University Press, Cambridge, 1977.

[41] Y.-D. Lenn and T. K. Chereskin, "Observations of Ekman Currents in the Southern Ocean," Journal of Physical Oceanography, Vol. 39, No. 3, 2009, pp. 768-779. doi:10.1175/2008JPO3943.1

[42] R. R. Schudlich and J. F. Price, "Observations of Seasonal Variation in the Ekman Layer," Journal of Physical Oceanography, Vol. 28, No. 6, 1998, pp. 1187-1204. doi:10.1175/1520-0485(1998)028<1187:OOSVIT $>2.0 . C$ $\underline{\mathrm{O} ; 2}$

[43] J. Heinloo and Ü. Võsumaa, "Rotationally Anisotropic Turbulence in the Sea," Annales Geophysicae, Vol. 10, 1992, pp. 708-715.

[44] Ü. Võsumaa and J. Heinloo, "Evolution Model of the Vertical Structure of the Active Layer of the Sea," Journal of Geophysical Research, Vol. 101, No. 11, 1996, pp. 25635-25646. doi:10.1029/96JC01988
[45] J. Heinloo and A. Toompuu, "Antarctic Circumpolar Current as a Density-Driven Flow," Proceedings of the Estonian Academy of Sciences, Physics and Mathematics, Vol. 53, No. 4, 2004, pp. 252-265. doi:10.1029/96JC01988

[46] J. Heinloo and A. Toompuu, "Modeling a Turbulence Effect in Formation of the Antarctic Circumpolar Current," Annales Geophysicae, Vol. 24, No. 12, 2006, pp. 3191- 3196.

[47] T. Whitworth, W. D. Nowlin Jr. and S. J. Worley, "The Net Transport of the Antarctic Circumpolar Current Trough Drake Passage," Journal of Physical Oceanography, Vol. 12, No. 9, 1982, pp. 960-971.

doi:10.1175/1520-0485(1982)012<0960:TNTOTA $>2.0 . C$ $\underline{\mathrm{O} ; 2}$

[48] J. Heinloo and A. Toompuu, "Modeling of Turbulence Effect in Formation of Zonal Winds," The Open Atmospheric Science Journal, Vol. 2, 2008, pp. 249-255.

[49] A. H. Oort, "Global Atmospheric Circulation Statistics, 1958-1973," NOAA Professional Paper 14, Rockville, 1983

[50] J. Heinloo and A. Toompuu, "Gyration Effect of the Large-Scale Turbulence in the Upper Ocean," Environmental Fluid Mechanics, Vol. 12, No. 5, 2012, pp. 429438. doi:10.1007/s10652-012-9247-2

[51] A. Toompuu and J. Heinloo, "Gyration Effect Estimated from Global Surface Drifter Data in the Pacific Ocean," IEEE/OES Baltic International Symposium, Klaipeda, 810 May 2012, pp. 1-4.

[52] A. L. Sybrandy and P. P. Niiler, "WOCE/TOGA Lagrangian Drifter Construction Manual. WOCE Rep. 63," Scripps Institution of Oceanography, La Jolla, 1991.

[53] A. Toompuu, J. Heinloo and T. Soomere, "Modelling of the Gibraltar Salinity Anomaly," Oceanology, Vol. 29, No. 6, 1989, pp. 698-702.

[54] R. W. Griffiths, E. J. Hopfinger, "The Structure of Mesoscale Turbulence and Horizontal Spreading at Ocean Fronts," Deep Sea Research Part A. Oceanographic Research Papers, Vol. 31, No. 3, 1984, pp. 245-269. doi:10.1016/0198-0149(84)90104-3

[55] J. Heinloo and A. Toompuu, "Eddy-to-Mean Energy Transfer in Geophysical Turbulent Jet Flows," Proceedings of the Estonian Academy of Sciences, Physics and Mathematics, Vol. 56, No. 3, 2007, pp. 283-294.

[56] P. B. Rhines and W. R. Holland, "A Theoretical Discussion of Eddy-Driven Mean Flows," Dynamics of Atmospheres and Oceans, Vol. 3, No. 2-4, 1979, pp. 289-325. doi:10.1016/0377-0265(79)90015-0

[57] J. R. Herring, "On the Statistical Theory of Two Dimensional Topographic Turbulence," Journal of the Atmospheric Sciences, Vol. 34, No. 11, 1977, pp. 1731-1750. doi:10.1175/1520-0469(1977)034<1731:OTSTOT $>2.0 . \mathrm{C}$ $\underline{\mathrm{O} ; 2}$

[58] F. Webster, "Measurements of Eddy Fluxes of Momentum in the Surface Layer of the Gulf Stream," Tellus, Vol. 17, No. 2, 1965, pp. 239-245.

[59] J. Heinloo, "Eddy-Driven Flows over Varying Bottom Topography in Natural Water Bodies," Proceedings of the 
Estonian Academy of Sciences, Physics and Mathematics, Vol. 55, No. 4, 2006, pp. 235-245.

[60] D. B. Haidvogel and D. H. Brink, "Mean Currents Driven by Topographic Drag over the Continental Shelf and Slope," Journal of Physical Oceanography, Vol. 16, No. 12, 1986, pp. 2159-2171.

doi:10.1175/1520-0485(1986)016<2159:MCDBTD $>2.0$. $\mathrm{CO} ; 2$
[61] F. P. Bretherton and D. B. Haidvogel, "Two-Dimensional Turbulence above Topography," Journal of Fluid Mechanics, Vol. 78, No. 1, 1976, pp. 129-154. doi:10.1017/S002211207600236X

[62] S. T. Adcock and D.P. Marshall, "Interactions between Geostrophic Eddies and the Mean Circulation over LargeScale Bottom Topography," Journal of Physical Oceanography, Vol. 30, No. 12, 2000, pp. 3232-3238. 